\title{
XTCE and XML Database Evolution and Lessons from JWST, LandSat, and Constellation
}

\author{
Jonathan Gal-Edd ${ }^{1}$ \\ GSFC/NASA, Greenbelt, Maryland, 20771, US \\ Stephen Kreisler ${ }^{2}$ \\ Honeywell/NASA, Greenbelt, Maryland, 20771, US \\ Curtis Fatig ${ }^{3}$ \\ SAIC/NASA, Greenbelt, Maryland, 20771, US \\ and \\ Ronald Jones ${ }^{4}$ \\ ASRC Aerospace/NASA, Greenbelt, Maryland, 20771, US
}

\begin{abstract}
The database organizations within three different NASA projects have advanced current practices by creating database synergy between the various spacecraft life cycle stakeholders and educating users in the benefits of the Consultative Committee for Space Data Systems (CCSDS) XML Telemetry and Command Exchange (XTCE) format.
\end{abstract}

\section{Introduction}

\footnotetext{
$\mathrm{T}$
} he James Webb Space Telescope (JWST) is a large aperture infrared space telescope with a five-year mission and ten-year design goal. It is currently scheduled to launch in 2013 from Kourou, French Guiana aboard an Ariane 5 launch vehicle. JWST is designated to succeed the Hubble Space Telescope (HST) as part of the National Aeronautics and Space Administration (NASA) Great Observatories program. JWST will continue the HST tradition of advancing breakthroughs in our understanding of the origins of the earliest stars, galaxies, and the elements that are the foundations of life.

The importance of operations in the mission lifecycle is sometimes overlooked during the development phase. Due to the long duration of JWST from over 20 years of development until endof-life the project has made a conscious decision to involve operations personnel from the beginning. JWST system engineers gathered in 2000 to look at various options, technologies, and industry trends to determine how to implement this objective. The JWST operations team performed studies to

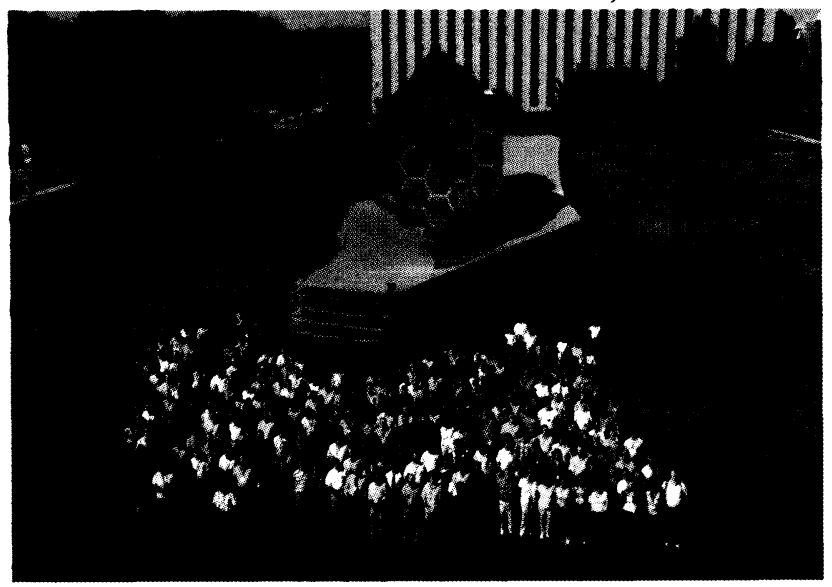

Figure 1. JWST Team. implement best practices and lessons learned from other GSFC long-term missions, such as HST and Earth Observation System (EOS). The major key decisions for

\footnotetext{
${ }^{1}$ TDRS-K Ground System Manager, SN, GSFC/Code 581, Greenbelt, MD, 20771, US, non-member.

${ }^{2}$ LandSat Database Manager, LandSat, GSFC/Code 400, Greenbelt, MD, 20771, US, non-member.

${ }^{3}$ JWST GS V\&V Manager, JWST, GSFC/Code 443, Greenbelt, MD, 20771, US, member.

${ }^{4}$ JWST Lead System Engineer, JWST, GSFC/Code 443, Greenbelt, MD, 20771, US, non-member.
} 
operations were; use of Consultative Committee for Space Data Systems (CCSDS) standards and institutional capabilities, use of the same command and telemetry system for I\&T and operations, and decouple the project reference database from the command and telemetry system. The JWST ground system is based on EOS and HST heritage and the lessons learned from these large scale missions. For HST, as systems were upgraded data conversion to a newer format became a major issue. For EOS, the early real-time ground system was not ready to support operations and was replaced by COTS system that was to be used for real-time operations. JWST is using the Raytheon Eclipse system for operations as well as for its software and hardware development and I\&T activities. Some of the major JWST enhancements to EOS version of Eclipse include providing a fast turn around (minutes) of database updates and storing the engineering data in a generic decommuntated format. A departure from both HST and EOS is that JWST is consolidating the science and mission operations.

The Landsat Data Continuity Mission (LDCM), shown in figure 2, is scheduled to launch in July 2011 and will capture space-based land remote sensing images consistent with predecessor Landsat satellites. The LDCM will provide repetitive acquisition of high resolution multi-spectral data of the Earth's surface on a global basis. The data from the Landsat series of satellites constitute the longest record of the Earth's continental surfaces as seen from space and are a valuable resource in the study of agriculture, education, business, science, and government. In order to reduce the effort to coordinate modifications to ground system databases seen in previous NASA missions, the LDCM project made a decision during the development of ground system requirements to maintain an integrated project reference database of all configuration managed data necessary to develop, test, and operate the Mission Operation Center (MOC). Like JWST, LDCM is consolidating it's science and mission operations in the United Stated Geological Survey (USGS) Sioux Falls, South Dakota facility. The LDCM Project will have a common database for I\&T and the operational system. LDCM, however, started from an initial baseline using the JWST

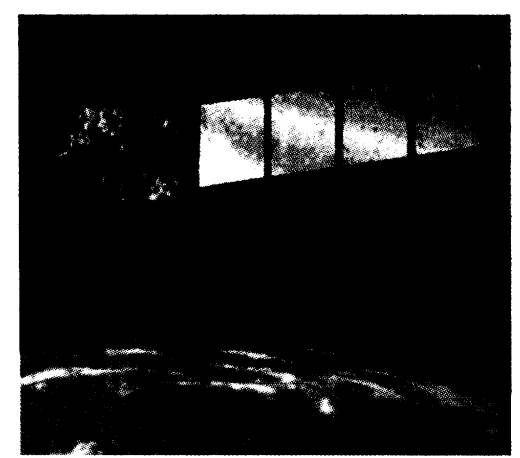

Figure 2. LandSat. XML database and CCSDS XML Telemetric and Command Exchange (XTCE) to develop its own database structures and tools.

NASA has formed the Constellation Program, Orion spacecraft shown in figure 3, to maintain American presence in low Earth orbit, returning to the moon for purposes of establishing an outpost, and laying the foundation to explore Mars and beyond. The Constellation Program's heritage rests on the successes and lessons learned from NASA's previous human spaceflight programs: Mercury, Gemini, Apollo, Space Shuttle and International Space Station (ISS). Constellation define its operation architecture in Command, Control, Communications and Information (C3I) specification. Realizing the Constellation needs to support multiple locations, such as Marshall Space Flight Center (MSFC), Johnson Space Center (JSC), and Kennedy Space Center (KSC), and multiple users services like the lunar surface, lunar orbiting satellites, International Space Station and earth, the C3I defines the operability specification for connecting this different diverse systems. Constellation C3I maximizes the uses of standards and it will be the first major NASA netcentric I- based system. Constellation will have a distributed science and mission

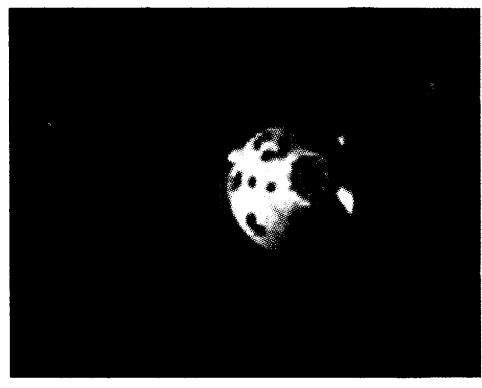

Figure 3. Constellation. operations, where KSC, JSC, and MSFC each have their own command and telemetry systems, analysis tools, and databases. This diversity in the Constellation Program means a consistent mechanism for moving the databases and products between different vendors, suppliers and international partners as needed. Since Constellation Program will mature for several decades the flexibility of XML and the definition of CCSDS XTCE will help achieve the goal of providing databases to dissimilar systems.

The focus of this paper is the need for all three of these long term programs to have databases to last a decade or longer for I\&T activities, mission support, and data processing. Also these three programs have international partners and a diverse user community for supplying equipment. CCSDS XTCE uses XML format and can provide a means of sharing the core database information in a consistent non-proprietary format. 


\section{The Advantages of Stand Alone Database using XML}

Control centers that support multi-missions and long term modern missions both at the European Space Agency (ESA) and NASA, recognize that moving and maintaining databases between dissimilar systems is risky and costly. While some Commercial-Off-the-Shelf (COTS) products may reduce a mission's development and software support costs, for missions of long duration that benefit might be reduced due to non-backward compatible software updates or elimination of feature support. To eliminate vendor dependency and reduce licensing cost JWST decided to use Open source XML rather than traditional data base programs.

XML allows for the data to be stored in a manner that allows it to be easily transformed, ingested, and accessible to many other systems. A sample of the database XML is shown in figure 4. The following paragraphs will describe each program's experience.

\section{A. JWST Program}

In 2001, the JWST database team started prototyping the JWST XML using the Earth Observation System (EOS) satellite database. This database contains about 25,000 data items for command and telemetry, which is the expected size of the JWST database. Once the database was converted from a COTS format to the XML, it took one person two weeks to complete the first steps in evaluating XML for performance, sizing, and exchanging the data between systems. The data is presented in the COTS in a typical user friendly web view. This web view, as shown in figure 5, hides the complexity of the XML data and with style sheets provides the first level of checking performed on the data. It is important to begin checking the data as the user enters it, so problems are found early and in a location that is easily corrected by the user. XML is the data at the bottom of the structure that is interpreted by the applications that presents the data to the user, see figure 6 .

The performance testing showed that database conversions to multiple formats could be accomplished in minutes using a standard desktop PC running Windows. For the JWST database each command and telemetry mnemonic would be a separate container or file. For example, if there are 200 commands in the database, then 200 containers would be delivered in a command folder. The sizing testing indicated no issues with each command and telemetry file about $4 \mathrm{~KB}$ in size, for a total of 100MB. The user interfaces were developed using a few key goals. The first goal is to use standard web browser interfaces so any workstation can use the database interface without having special software. The second goal is to remove any of the XML tags and code from the

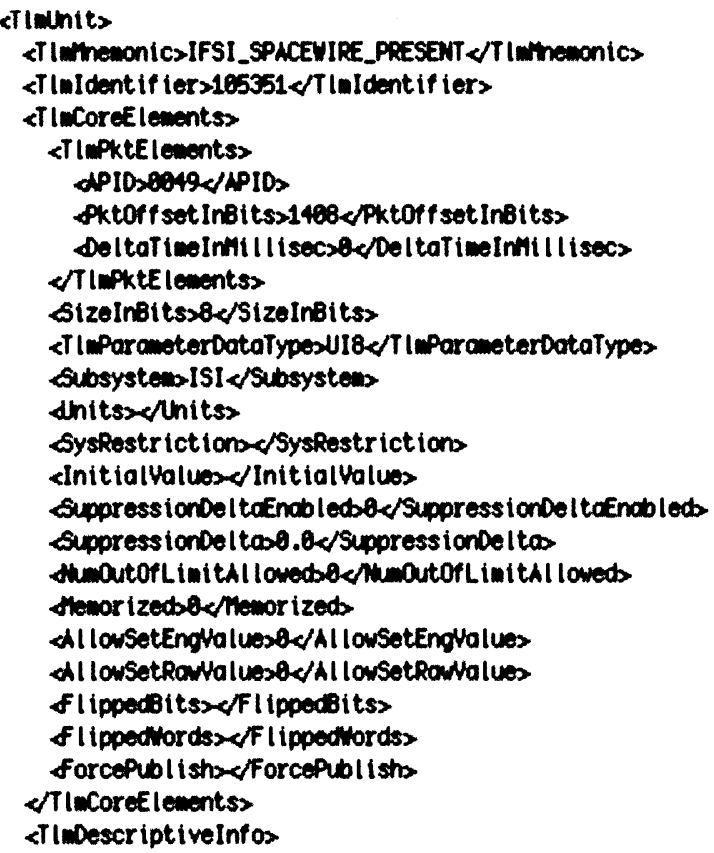

Figure 4. XML Sample.

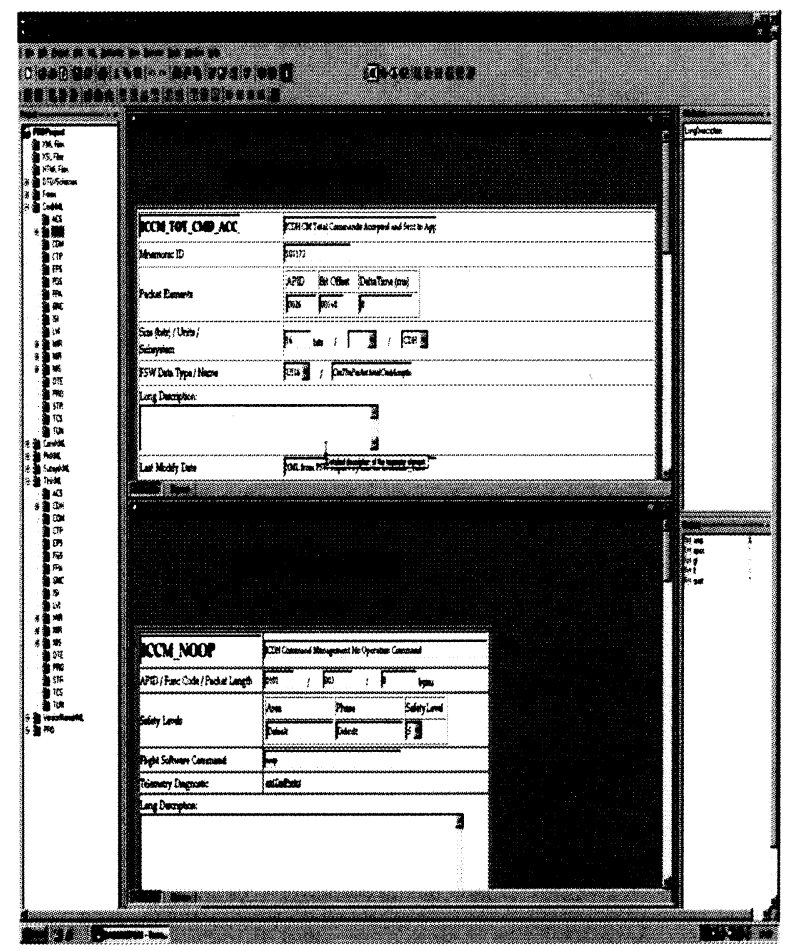

Figure 5. User Display. 
user experience. The third and final goal, the input form is to check for legal values to identify as many errors as far forward in the process as possible to reduce the amount of re-work the user would need to do.

The JWST database is currently one of the largest XML spacecraft databases for the real-time and offline systems at Goddard Space Flight Center. The current database system is available to 34 distinct laboratories, each geographically dispersed, having local database tools to work with the XML databases. Each of these laboratories database tools are used for the exporting and importing of data both locally and to the central database system, inputting data to the database certification process, and

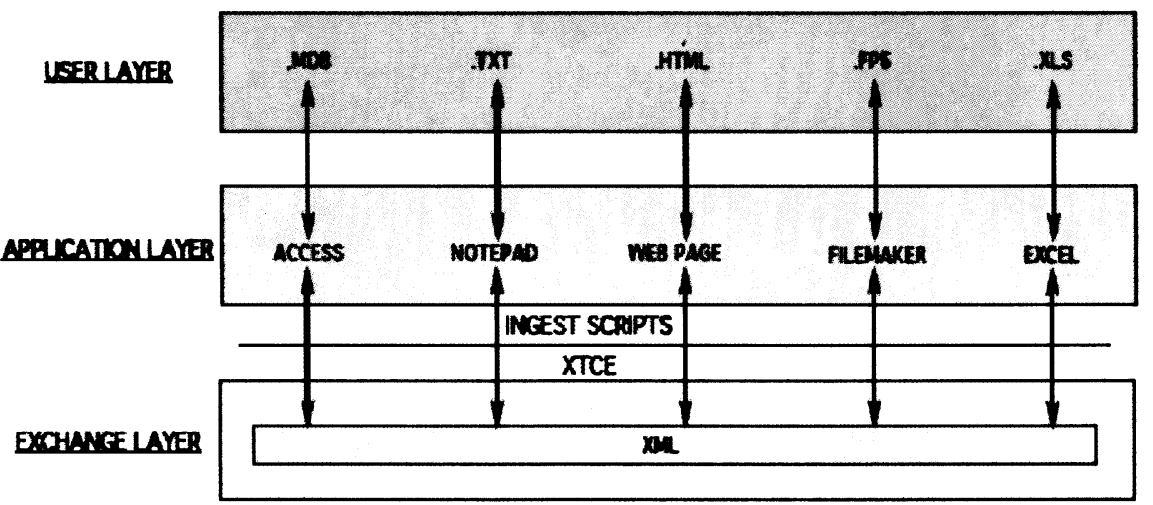

Figure 6. XML Layers. providing various reports.

\section{B. LDCM Program}

The decision by LDCM management to maintain a centralized project reference database was made early in 2007 , prior to the selection of instrument, spacecraft, and ground system vendors (as of this writing, only the instrument vendor has been selected). As part of this decision, the project evaluated the JWST design and concluded that its XML data exchange format was appropriate for LDCM. The decision was based on a number of factors, including the application independent, ASCII-based structure of XML, the compatibility with the CCSDS XTCE standard, and the proven performance of the JWST system. Like JWST, LDCM will gather database contributions from external laboratories, albeit on a smaller scale. While many COTS product are currently able to import XML structures, the utility of Extensible Stylesheet Language Transformations (XSLT) allows external users who cannot directly ingest XML into their applications to convert the database to an acceptable format. The reduction in cost, associated with the non-proprietary and flexible transformation characteristics of XML, in addition to the minimization of risk, due to the commonality of database field interpretation taken from the XTCE standard, further substantiated the appropriateness of XML as the database format.

\section{Constellation Program}

The Constellation Program C3I specification looked for standard ways of defining it's interfaces. C3I uses CCSDS XTCE as a means to describe the database as well as provide an exchange method . The experience of many of the Constellation personnel are from the NASA manned flight programs, including the space shuttle and International Space Station. The experience of these personnel meant that changes occurred very slowly and methodically and with the introduction of CCSDS XTCE very few Constellation personnel with little real world experience with XML. At the 2007 CCSDS committee meeting in the Spacecraft Monitor and Control working group, the Constellation and XTCE personnel took an existing space shuttle database and provided a comparable CCSDS XTCE database in about 10 hours. Based on this effort it was felt that using CCSDS XTCE as both the mission command and telemetry database as well as exchange formats was entirely feasible. The Constellation Project continues to evolve the databases needed to support the various systems and elements using XML and CCSDS XTCE where applicable. With the launch dates for Ares and Orion coming closer, Constellations is redefining the portions of it's C3I information model to focus on command and telemetry using XTCE.

\section{Evolution of CCSDS XTCE Standard}

Declaring XTCE as an open standard exchange format and what this means has been one of the challenges. JWST personnel first heard of the emergence of XTCE as a combined OMG/CCSDS standard at a conference in 
2004. A draft of the proposed XTCE standard was presented and JWST personnel had concerns that the current implementation of the JWST database would not confirm with the proposed standard and would violate a JWST project requirement to be compatible with the CCSDS standard. Due to the JWST commitment to conform with standards, JWST personnel started meeting with various industry standard groups, such as the OMG and the CCSDS Spacecraft Monitor and Control Working Group, to ensure that JWST XML maintained a level of compatibility with the draft standard and assisted in writing of the CCSDS XML standard. Working with the standards was especially challenging for a pure CCSDS project like JWST, as the standard was trying to address both TDM and CCSDS users, that complicated the standard with what JWST considered 'extra' tags.

In helping to develop the standard, the JWST Project took the role of coordinating with the developers of Integrated Test and Operations System (ITOS), Advanced Spacecraft Integration and System Test (ASIST) command and telemetry systems, and the JPL Mars mission teams to provide a level of consistency among the various NASA projects and to build consensus for the CCSDS standard. One of the common complaints about the proposed standard was its complexity and this issue was taken into account by the CCSDS standards group. JWST and JPL personnel met several times and after reviewing all the XML tags and structures, it was discovered that the JWST XML database is a subset of the JPL XML database. The two different XML databases, designed independently from one another, were found to be about $95 \%$ compatible with other on commands and $90 \%$ compatible on telemetry without any changes to either database. When some minor additions were made to the JPL XML, the compatibility increased to $100 \%$ for commands and $95 \%$ for telemetry.

JWST personnel took the inputs from the various NASA groups mentioned above and began

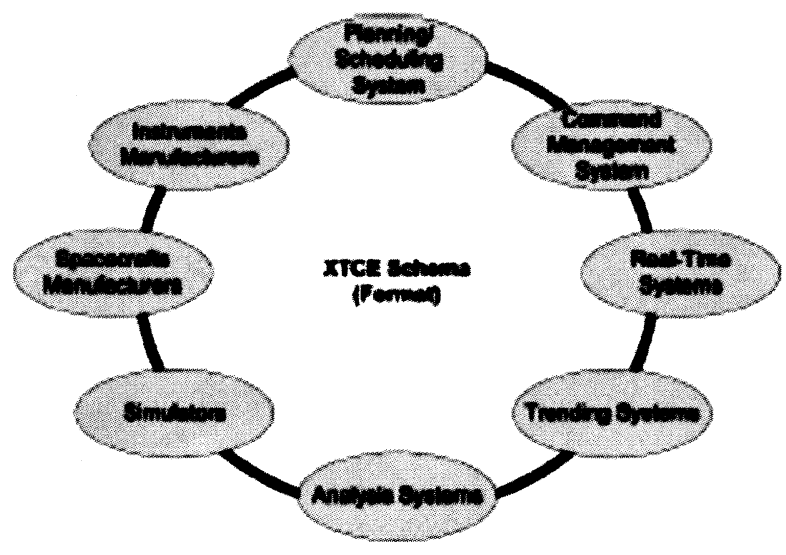

Figure 7. CCSDS Green Book XTCE Exchange Concept. working with OMG and CCSDS members to address the complexity of the CCSDS XML standard. The two main contributors to developing the XTCE standard working group were ESA/ESOC and NASA/JWST. To help deal with the complexity of XTCE the CCSDS XTCE working group developed the 'green book' as a user's guide for the various managers and users not familiar with XML to understand the purpose and structure of the CCSDS XML database standard, as shown in figure 7 . The 'magenta book' was developed to assist the XML database developers by providing examples, alternative ways of using the CCSDS XML tags, and a means to incorporate database items that are not covered under the CCSDS standard, as shown in figure 8.

From the perspective of the JWST Project, the experience of working with the CCSDS standards group was informative in learning the way standards are developed, provided a greater appreciation for the amount of time and effort it takes to get agreements on a standard, and also provided stimulating interaction with dedicated and intelligent personnel from the various international groups.

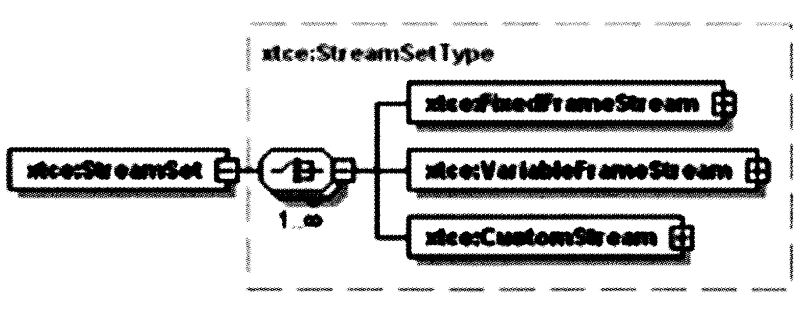

Figure 8. CCSDS Magenta Book Sample. 


\section{Beyond XTCE : Tools and Processes}

The success of JWST database is, that in addition to XML and XTCE, it provides tools and processes. These tools have been refined during 4 years of experience in the I\&T environment.

- The command and telemetry CCSDS XTCE database standard is only one piece of the entire satellite project puzzle. JWST uses XML for display pages, science processing, loads and dumps, engineering data requests, and several other products needed for the entire JWST mission.

- Add XML tags for Configuration management and internal documentation. This will allow for creating software generated reports and auditing.

- Include the database process early at the developer site provides a great benefit in identifying the problems early. Prepare an Interface Control Document (ICD) early in the process that describes the data needed, allowed values, and any parent/child relationships.

- For some missions are requiring the developers to deliver the database with the product in CCSDS XTCE format, others convert to XTCE after receiving the data, and finally some missions provide tools to the developer to assure the database is the same at I\&T as well as the mission. No one way is better than the other, but chose one process then developed the rules, interface control document, and tools necessary before releasing to the users.

- Do not change tag name. The changing of tag names will have a ripple effect on any software that is used to translate the XML into the systems native format. It is often hard to know all the places where the tag names are used. The more appropriate means is to add new tags and to retire tags no longer needed.

- Do not change directory names. As stated above changing the directory name will have the same impact as changing the tag names. However, adding and retiring directory names creates issues as well. If needing to change do so very infrequently. In general most users of the systems that ingest the database never see the lower level database directory structure, so changing the directory name has little value for the end user.

- Decide early one or many data structure. This data structure will determine the various tools and relationships that will be needed to support the database.

- Provide a database tool kit to the developers. This tool kit provides the view of the database to the end user and other tools for checking of the data, submitting of the data and downloading golden databases from a central site. The JWST tool kit flow that is used at the developer sites and I\&T facilities is shown in figure 9. 


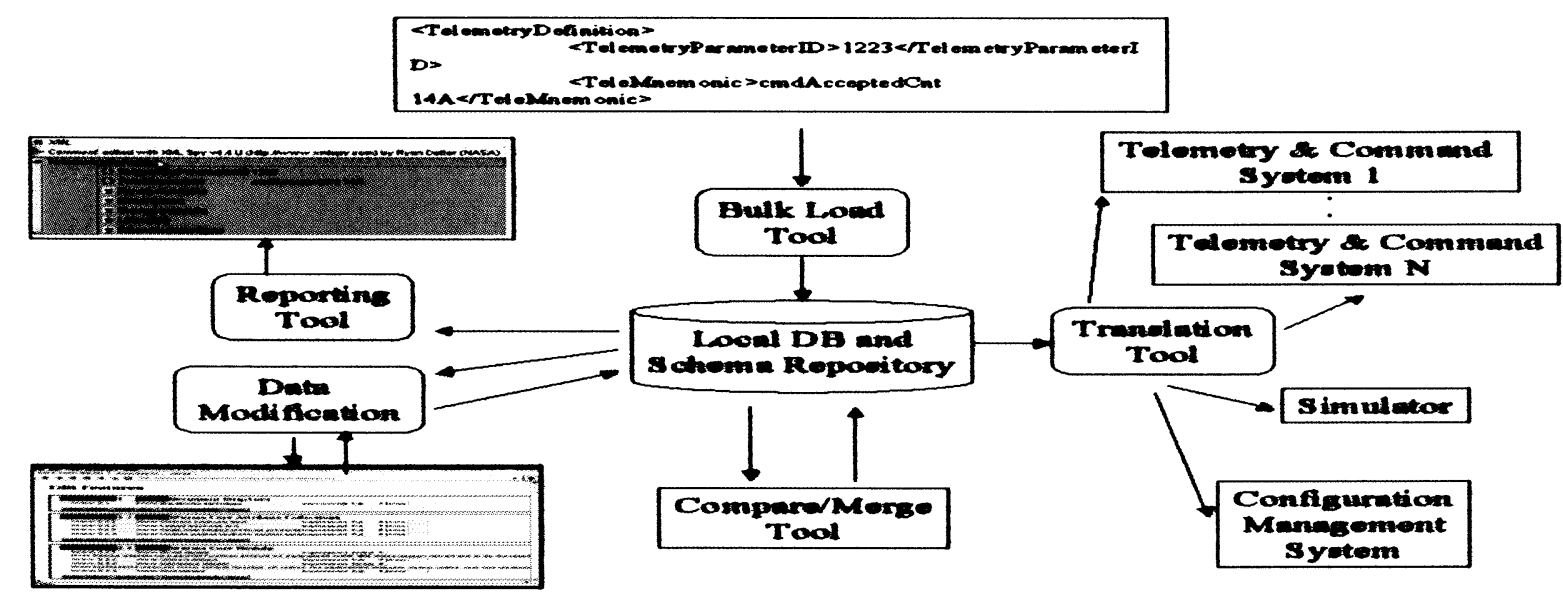

Figure 9. Integration and testing Database Tool.

- Provide conversion tools when adding tags, directories, or required values. The conversion tool, as a stand alone piece of software, can convert all the data quickly. A report should be produced that shows what items have been added or changed so the user can review. If possible the conversion tool should also modify the other database products such as scripts and pages.

- Include the schema checks in the user toolkit to catch problems early. The style sheets and schemas allow for pick lists, range checks, naming rules, and other helpful information so that user input conforms to the ICD definitions. This will save rework by the end user and reduce the frustration that often occurs.

- Include the schemas in the database itself, this will allow for the checks to be updated without effecting previous databases where the checks were not enforced.

- Support Multiple database types; test database for future releases, golden database for current users, and an area for incomplete data.

- Verification reports that check all the content to the database ICD at the user site and the central database group. These verification reports often call on other software to do more complex checking that can not be easily done with schemas. The verification reports can be used to correct errors prior to submitting the data to the central database group.

- Allow for the database ICD and tags to mature. Since the database is needed early in I\&T and as the project moves through the various development phases, the data will mature. This maturing data will require changes so database groups should plan on it.

- Allow the users to quickly make new database and merge new database with an existing database. In I\&T it is not uncommon to have 10 or more databases a day. Also to prevent the natural habit of changing the flat files, the database creation should be in the 5 minute or quicker timeframe.

I\&T database tool needs are far more demanding then normal operations, but the investment in the tools and creating the data in the proper format is well worth it in the end.

\section{Lessons of XML and CCSDS XTCE}

XML provides many benefits such as long-term support, non-proprietary format and is easily convertible to existing applications. These database efforts for the three programs are still ongoing, but the following has been noted: 
- Translating a mission XML database to XTCE is not difficult.

- A common mission XML format between dissimilar systems is possible.

- A common tool set for viewing and organizing the XML and XTCE is useful.

- Command XML and XTCE is more complex than telemetry.

- For non-XTCE data, the use of XML metadata to describe item such as pages and scripts was needed due to the proprietary nature of most current ground systems.

Understanding that XTCE was designed as an exchange format and not a mission database is key. Although XTCE can be a mission database for the command and telemetry structures, there are many other pieces needed to make a complete database.

XTCE and XML in general are read by computer systems, not people, so only the programmers need to understand XTCE/XML tags. The users of the data use a COTS or a web interface that will present the data in a user friendly manner.

\section{Summary}

The combination of XML for managing program data and CCSDS XTCE for exchange is a robust approach that will meet all user requirements using Standards and Non proprietary tools.

COTS tools for XTCE/XML are very wide and varied. To combine together various low cost and free tools can be more expensive in the long run than choosing a more expensive COTS tool that meets all the needs. This was especially important when deploying in 32 remote sites with no need for licenses.

A common mission XTCE/XML format between dissimilar systems is possible and is not difficult. Command XML/XTCE is more complex than telemetry and the use of XTCE/XML metadata to describe pages and scripts is needed due to the proprietary nature of most current ground systems. Other mission and science products such as spacecraft loads, science image catalogs, and mission operation procedures can all be described with XML as well to increase there flexibility as systems evolve and change. Figure 10 is an example of a spacecraft table load.

The word is out and the XTCE community is growing, The first XTCE user group was held in October and in addition to ESA/ESOC, SCO2000, and CNES identified several systems based on XTCE. The second XTCE user group is scheduled for March 10, 2008 with LDMC and others joining. As the experience with XTCE grows and the user community receives the promised benefits of using XTCE and XML the interest is growing fast.

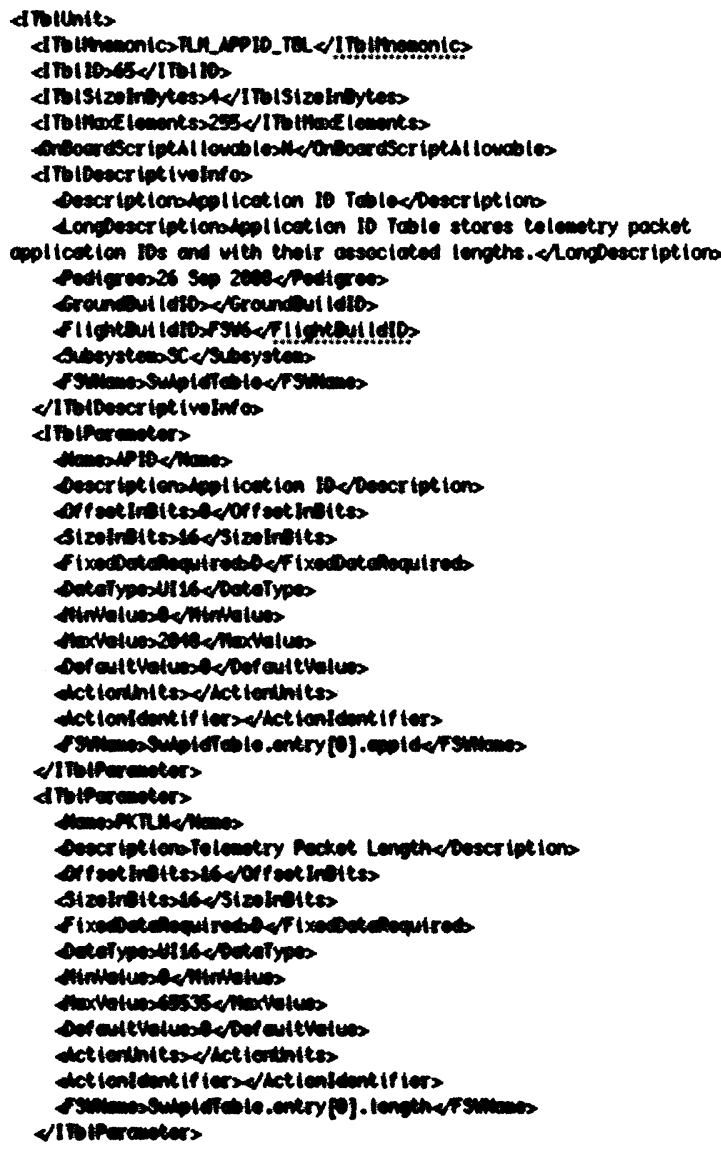

Figure 10. Table Example. 